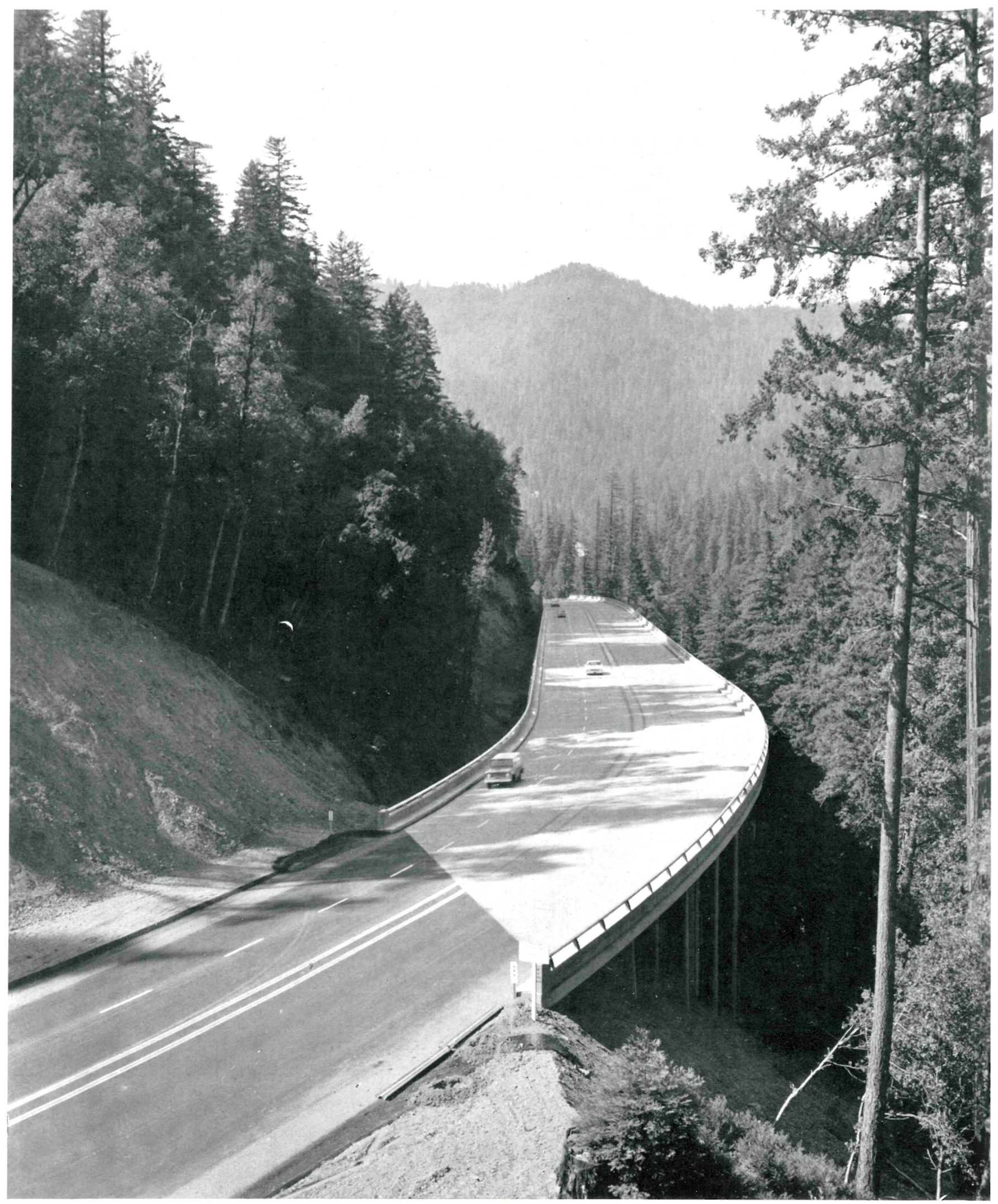

\title{
paso superior en una ladera
}

$569 \cdot 11$

O. BENDER, ingeniero 


\section{sํำ}

La autopista denominada Redwood (California) salva, con un paso superior, la ladera de un bosque cuya pendiente es del 1/1. El terreno firme se halla a bastante profundidad, añadiéndose, a los naturales problemas de la construcción, el imperativo de respetar la belleza agreste del paraje.

La solución adoptada consiste en una estructura porticada, con varios tramos de pequeñas luces, salvados con vigas metálicas, sobre los que se coloca la losa del tablero, de $19 \mathrm{~m}$ de anchura total. Los soportes están constituidos por pórticos de dos montantes huecos (con bases de hormigón en masa por debajo del suelo, hasta el firme) coronados por un cabezal.

La perforación de pozos para el hormigonado de los montantes presentaba la dificultad de atravesar el terreno superficial de acarreo y la de retener los escombros, con el fin de evitar daños a los troncos de los árboles.

El problema principal se centró en la ejecución, pues no se disponía de accesos amplios ni de plataformas apropiadas para las máquinas auxiliares, lo que motivó que gran parte del trabajo de cimientos fuera manual.

Como un borde del tablero se halla muy próximo a la ladera, en esta parte se construyó una viga de apoyo, de hormigón, sobre pilotes metálicos.

EI entramado-soporte de encofrados de la losa del tablero se apoyó en el cuerpo de hormigón de los montantes.

La ejecución de la autopista de Redwood, situada en la parte septentrional de California (Estados Unidos), ha presentado diferentes problemas $\mathrm{y}$, entre ellos, el que se refiere al paso sobre una ladera espesamente poblada de pinos de gran vuelo.

Era imprescindible no mancillar la belleza natural del lugar con la presencia de una obra de fábrica; tampoco debían talarse árboles y, finalmente, se debían conservar las curvaturas, tanto horizontales como verticales, dentro de los límites

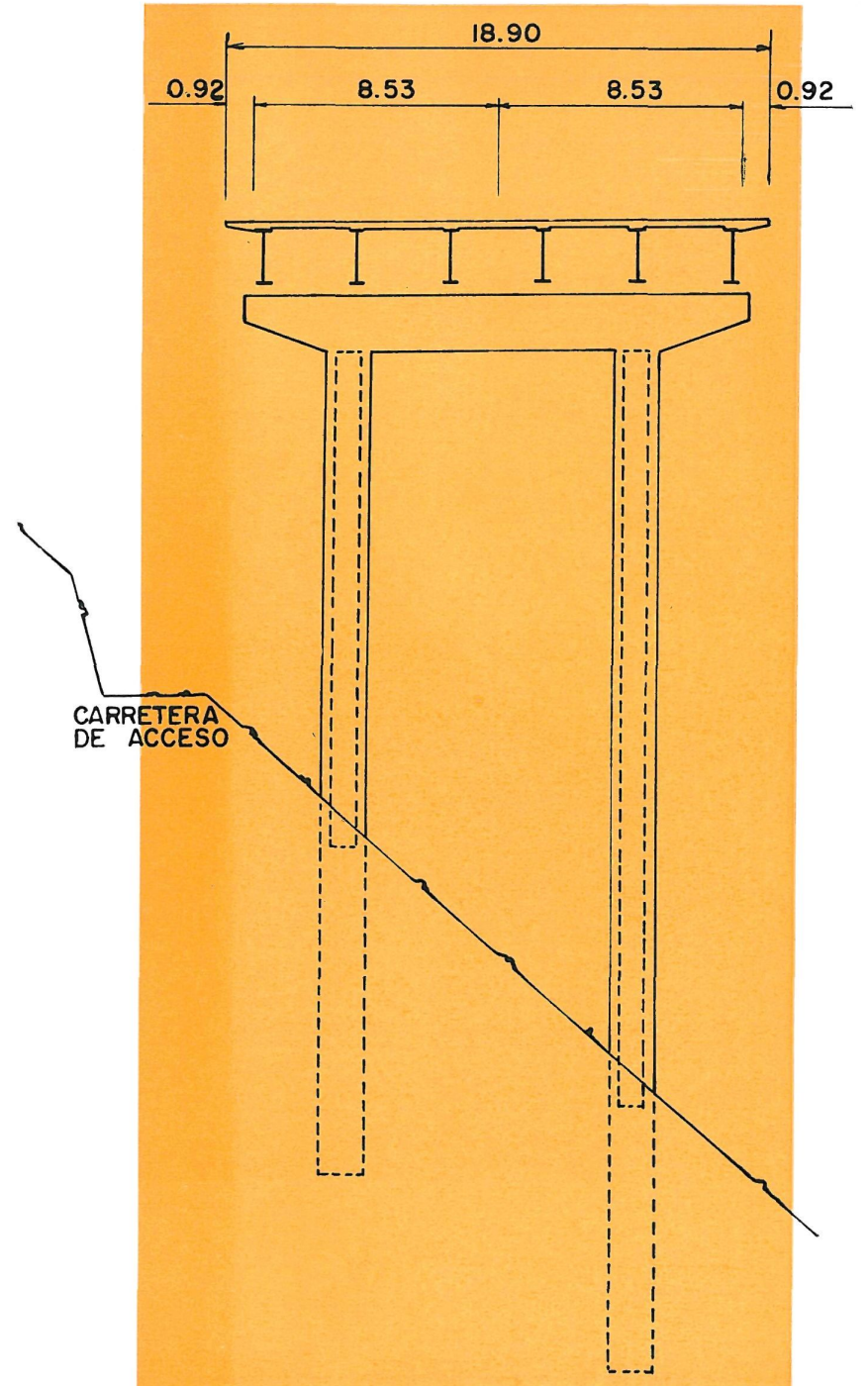

soportes y sección del tablero reservados a las bandas de circulación de este género especial de carreteras.

El denominado viaducto de Eagle Point se apoya sobre una ladera que discurre paralelamente a uno de los brazos del río Eel, que se encuentra a una cota unos $45 \mathrm{~m}$ por debajo de la correspondiente a la traza del eje sobre la ladera. La ladera de apoyo tiene una pendiente de $1 / 1$ y se halla cubierta de abetos y pinos. En algunas zonas claras se hace evidente que ha habido corrimientos.

Los sondeos previos de reconocimiento denotaron la existencia de una capa de 3 a $6 \mathrm{~m}$ de potencia, que se componía de detritus suelto y de unos 3 a $4,5 \mathrm{~m}$ de pizarras fracturadas, por debajo de las cuales aparecía un banco sano de esquistos o areniscas. Se dedujo que la capa de detritus, dada la gran pendiente, sólo se podía estabilizar mediante raíces arbóreas y algunos apuntamientos rocosos.

Por todo ello, y después de un detenido estudio, se llegó a la conclusión de que la estructura en cuestión, aparte de ser económica, tenía que ser levantada sin entramados auxiliares y los cimientos debían realizarse con excavaciones mínimas.

Dadas las condiciones locales, y teniendo en cuenta los precios del hormigón y acero, se eligió un tipo de vigas metálicas, armadas, que se creyó era la solución más viable. En efecto, resultó ser el 8 por 100 más económica que las estructuras tipo cajón hormigonadas en obra y el 12 por 100 más ventajosas que las vigas prefabricadas, de sección en doble $\mathrm{T}$ y pretensadas.

Hecha la elección se efectuaron una serie de cálculos, utilizando un computador electrónico, para determinar el tipo, canto y espaciamiento de las vigas que resultara más barato.

El mayor canto de la estructura es de $2,1 \mathrm{~m}$, subdividiéndose en once tramos de unos $33,5 \mathrm{~m}$ de luz cada uno. La pequeña variación de luz entre ellos se debe a que el trazado tiene una alinea. ción en curva. En una extremidad se han levantado cinco tramos continuos, de $8,5 \mathrm{~m}$ de luz cada uno, sobre los que se hormigonó una losa, de hormigón, apoyada sobre pilotes. Estos tramos tienen por objeto compensar un esviaje de $61^{\circ}$ en el estribo y algunas irregularidades del terreno. 


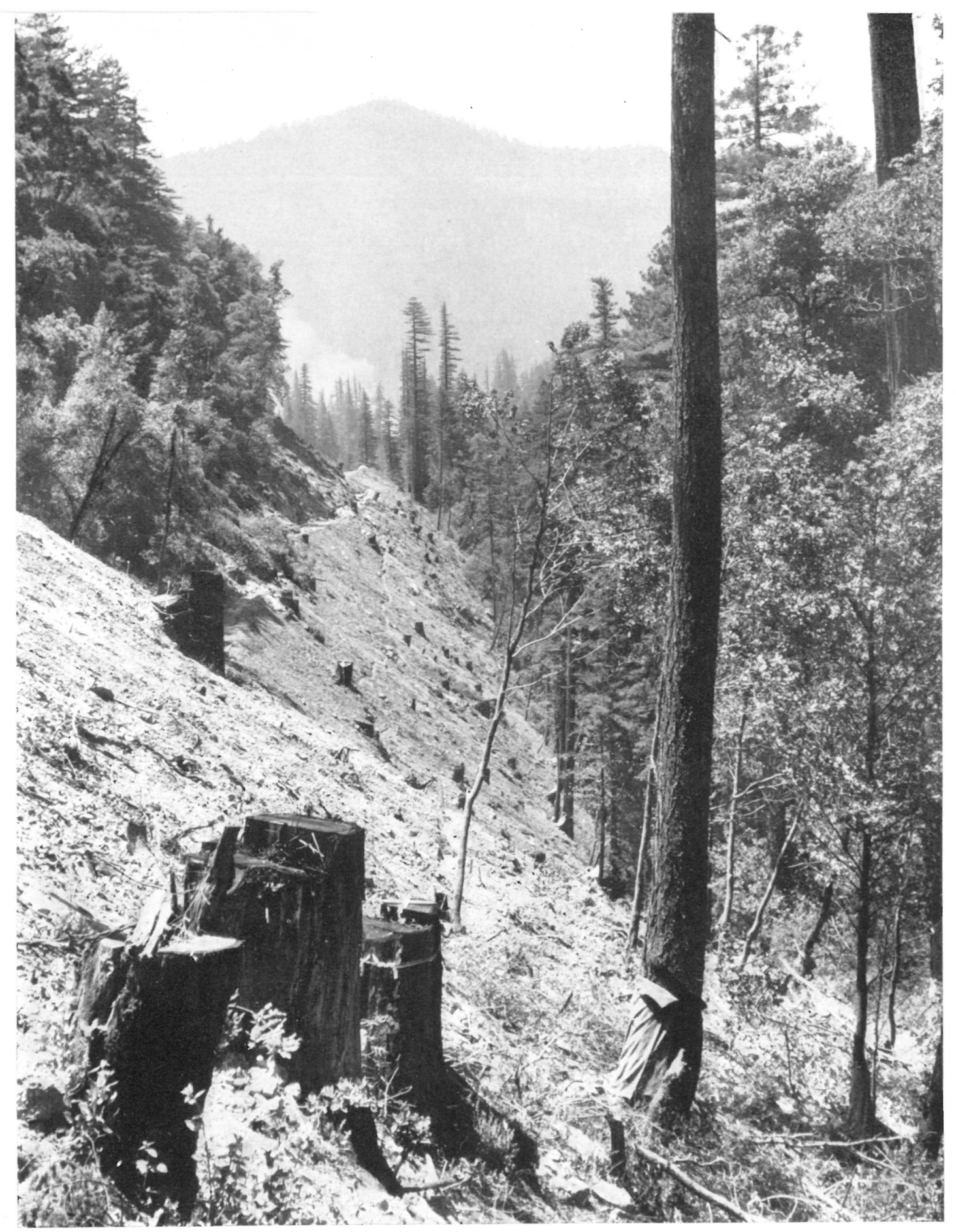

Tala inicial.

El tablero, de $19 \mathrm{~m}$ de anchura, se soporta con seis vigas metálicas, a excepción de los tres últimos tramos, en los que sólo se han colocado cinco vigas debido a la proximidad del terreno con el borde del tablero, circunstancia que se aprovechó para construir una viga de hormigón, apoyada sobre pilotes metálicos.

Los soportes de la estructura se componen de dos montantes, coronados con un cabezal formando pórtico, que penetran unos $3 \mathrm{~m}$ en el interior de un banco sano de esquistos o areniscas. Estos montantes tienen una sección, de $1,50 \times 1,50$, maciza por debajo del suelo y hueca en la parte superior.

Para dar acceso a materiales y maquinaria auxiliar se construyó un camino a media ladera, a una cota ligeramente superior a la rasante de la autopista. Los pocillos abiertos para hormigonar los montantes se excavaron con una perforadora de rotación, y en algunos de éstos se tuvo que preparar un entramado como plataforma de trabajo. Para la entubación de brocales se emplearon virolas metálicas en las zonas superficiales de tierras sueltas. Al llegar al firme, la perforación se ejecutó por procedimientos manua- 


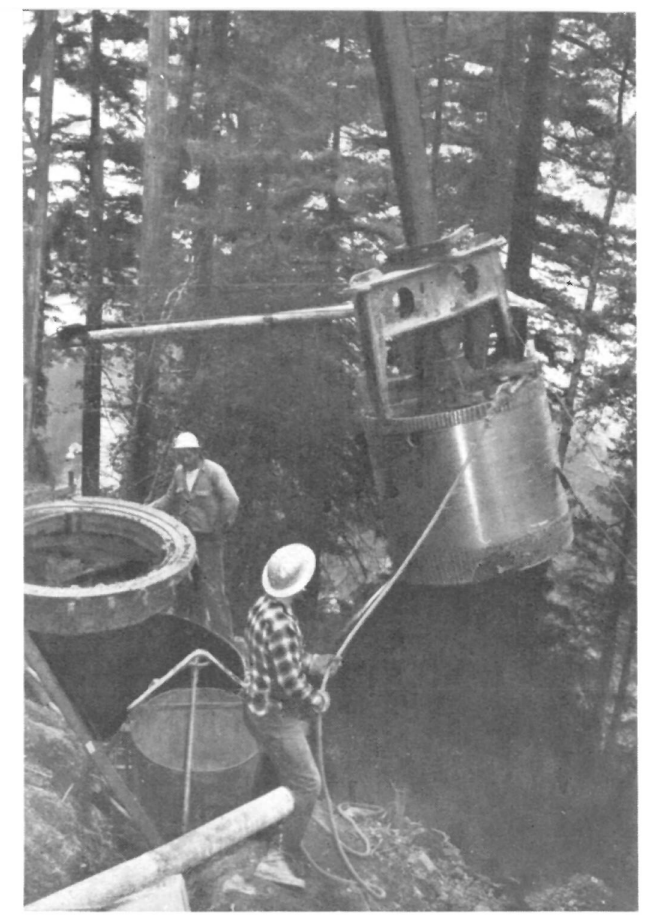

mo destos pocillos no pudieron excavarse mecánicamente y, cionales en las labores subterráneas.

Los pocillos se entibaron convenientemente para evitar avenidas de escombros.

Todos los montantes se apoyan, en su base, de 9 a $12 \mathrm{~m}$ por debajo del suelo natural y sobre un banco de roca firme. Los escombros de la perforación se tuvieron que apilar cuidadosamente para evitar pudieran rodar por la ladera hasta el río. Los pinos y abetos se protegieron apropiadamente, con objeto de evitar posibles daños en los troncos por impactos con cantos rodados procedentes de la excavación de pocillos. El montante de mayor longitud tiene $26 \mathrm{~m}$ de altura entre el suelo e intradós del cabezal.

Los encofrados para el hormigonado de este último se apoyaron sobre un entramado auxiliar levantado sobre los montantes.

La colocación de las vigas metálicas, que fue realizada con grúas que trabajaban a un lado del viaducto, presentó no pocos problemas a causa del reducido espacio de que se disponía; no obstante, y gracias al planeamiento cuidadoso de toda la obra, se pudieron vencer dichas dificultades y cumplir los plazos previstos.

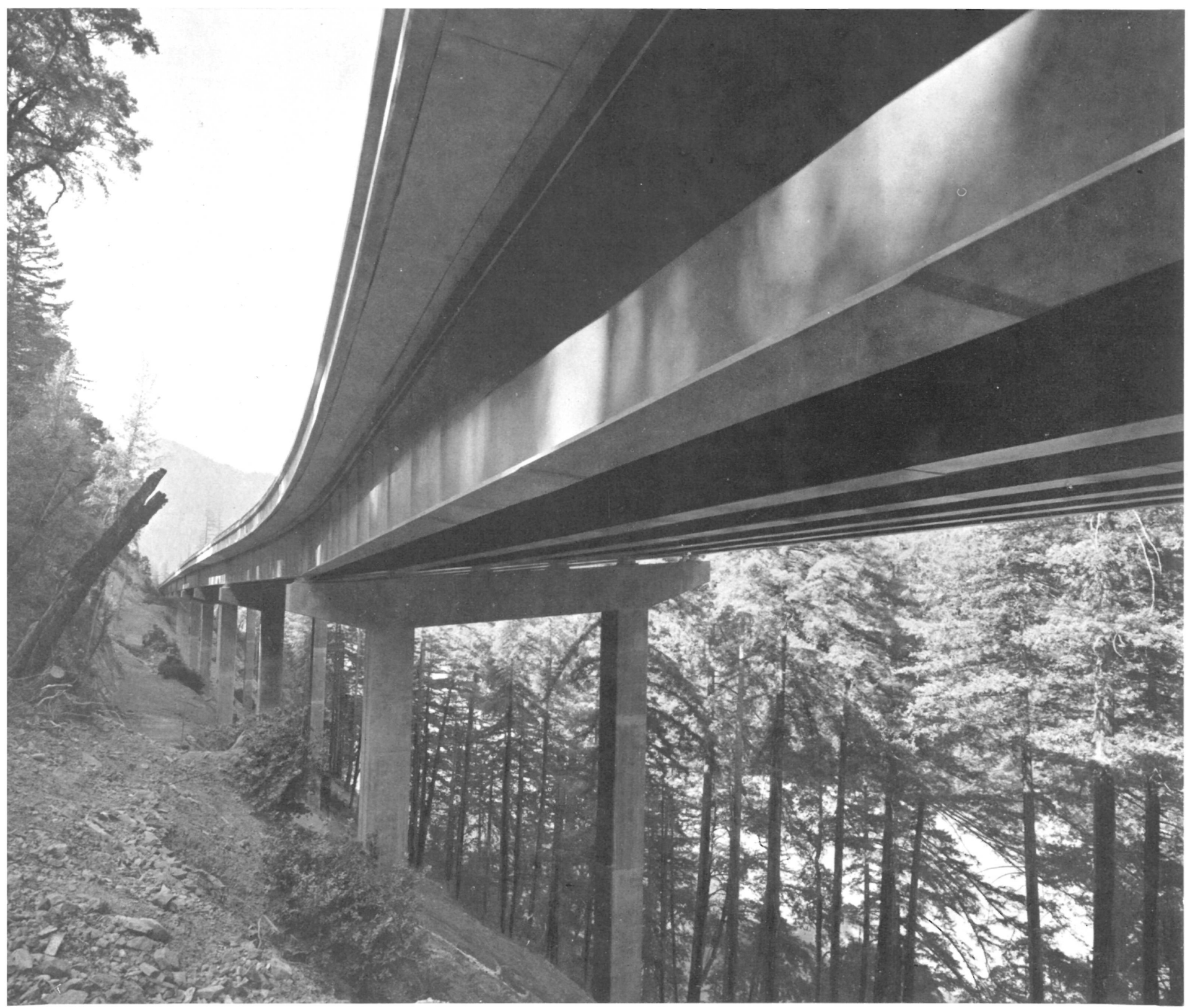




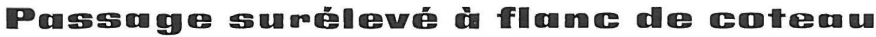

o. Bender, ingénieur.

L'autoroute du nom de Redwood (Californie) franchit, à l'aide d'un passage surélevé, une pente boisée dont l'indice est de 1/1. Le terrain solide se trouve à une certaine profondeur et l'obligation de respecter la beauté agreste de l'endroit s'ajoute aux problèmes naturels de la construction.

La solution adoptée consiste en une structure en portique, de plusieurs travées de faibles portées, franchies par des poutres métalliques, sur lesquelles est placée la dalle du tablier, de $19 \mathrm{~m}$ de largeur totale. Les supports sont formés par des portiques à deux montants creux (reposant sur des bases de béton en masse audessous du sol, jusqu'au terrain solide) couronnés par un croisillon.

La perforation des puits pour le bétonnage des montants présentait la double difficulté de traverser le terrain superficiel d'alluvion et de retenir les déblais, pour ne pas abîmer les arbres.

Le principal problème fut l'exécution, car ne disposant pas d'accès suffisants ni de plate-formes appropriées pour les engins auxiliaires, une grande partie du travail de fondations dut être effectuée à la main. Un des bords du tablier étant très proche de la pente, on a construit à cet endroit une poutre d'appui, de béton, sur pilotis métalliques. Les supports des coffrages de la dalle du tablier s'appuie directement sur une entaille prévue dans les montants.

\section{Roud aliomg ancumtaim side}

o. Bender, engineer.

The Redwood highway, through the Californian forest, runs on a viaduct, as it crosses a mountain slope of about $45^{\circ}$ inclination. The firm ground is fairly deep, and as an additional constructional difficulty, it was necessary to respect the natural beauty of the countryside.

A structure of portal frames were built, forming a number of short spans. These spans were bridged with metal girders, on which a $19 \mathrm{~m}$ wide deck was placed. The columns are hollow and have a transversal cross beam, to join each pair.

There was difficulty in excavating the foundations for the columns, as it was necessary to dig through the soft top soil, and also prevent this soil from hurting the trunks of the forest trees. Another significant difficulty in the construction of this viaduct was the access to the working site, since there were no suitable platforms from which to operate the appropriate machinery. This made it necessary to do a lot of the work by manual operation. As one of the edges of the deck is very close to the mountain side, a supporting beam was erected on this side. It was made of concrete, on metal piles. The formwork for the deck structure was placed on the concrete stems of the supporting piles.

\section{Hochstrusse an einem Hang}

o. Bender, Ingenieur.

Die Autobahn von Redwood (Kalifornien) überbrückt in Form einer Hochstrasse den Hang eines Waldes, der eine Neigung von $1 / 1$ besitzt. Der tragfähige Boden liegt sehr tief. $\mathrm{Zu}$ dieser Schwierigkeit kommen noch die allgemeinen Probleme hinzu, die ein Bau mit sich bringt und ausserdem die Forderung, die landschaftliche Schönheit zu respektieren.

Die ausgeführte Lösung besteht in einer Rahmenkonstruktion, mit mehreren Feldern kleiner Spannweiten, die mit Hilfe von Metallträgern überbrückt werden. Auf ihnen liegt die Fahrbahnplatte mit $19 \mathrm{~m}$ Breite. Die Brückenpfeiler sind Rahmen aus zwei Hohlstützen (mit Betonbasis unter der Bodenoberfläche) mit einem Riegel.

Die Bohrungen zum Einbetonieren der Pfosten hatten ihre Schwierigkeiten: Man musste die obere lockere Erdschicht durchqueren und die beim Bohren anfallende Erde so lagern, dass die danebenstehenden Bäume nicht geschädigt wurden.

Doch die Hauptschwierigkeit lag in der Ausführung, denn man verfügte weder über breite Zufahrtswege noch über geeignete Flächen zum Aufstellen der Baumaschinen, was zur Folge hatte, dass man einen grossen Teil der Fundamente mit der Hand machte. Da die eine Kante der Brückenplatte sehr nah am Abhang liegt, baute man dort einen Stützträger aus Beton, der auf Stahlstützen aufliegt. Das Schalenstützwerk der Brückenplatte liegt auf den Betonpfosten der Rahmen auf. 